\title{
INVESTIGATION OF PRACTICAL AND THEORETICAL ACCURACY OF WIRELESS INDOOR POSITIONING SYSTEM UBISENSE
}

\author{
Marek Woźniak, Waldemar Odziemczyk, Kamil Nagórski \\ Faculty of Geodesy and Cartography \\ Warsaw University of Technology \\ Warsaw, Poland
}

\begin{abstract}
This paper presents the accuracy investigation results and functionality of Ubisense RTLS positioning system. Three kinds of studies were conducted: test of calibration accuracy, analysis of theoretical accuracy of the coordinates determination as well as accuracy measurements in field conditions. Test of calibration accuracy was made with several different geometric constellation of reference points (tag positions). We determined changes of orientation parameters of receivers and disturbance of positioning points coordinates against chosen reference points constellations. Analysis of theoretical accuracy was made for several receivers spatial positions and their orientations. It allowed to indicate favourable and unfavourable measurement area considering accuracy and reliability. Real positioning accuracy of the Ubisense system was determined by comparison with coordinates measured using precise tacheometer TCRP1201+.

Results of conducted experiments and accuracy analysis of test measurement were presented in figures and diagrams.
\end{abstract}

Keywords: positioning systems, RTLS, point tracking

\section{Introduction}

The Indoor type spatial positioning systems are a particular group of positioning systems. They allow for the real time determination of spatial positions of humans, animals or other items inside closed rooms.

Such systems usually utilize electromagnetic waves of various frequencies. Applied positioning solutions are also different.

The Indoor type positioning systems are applied in many sectors of humans' activities, such as logistics, health care, sport, defense, breeding animals, or various 
industrial sectors. The Indoor systems may be very useful for various emergency operations, performed in conditions of threats for human health and life. The RTLS system developed by Ubisense is one of the Indoor type positioning systems.

\section{Principle of operation of Ubisense system}

The Ubisense RTLS (Real Time Location System) utilizes the UWB wavelength (ultra wide band - frequency between $6-8 \mathrm{GHz}$ ) and the additional channels of $2.4 \mathrm{GHz}$. Such a solution allows for effective elimination of noises caused by the signal multipath (Fig. 1). This problem was described by O. Gremigni and D. Porcino in (Gremigni \& Porcino, 2006).

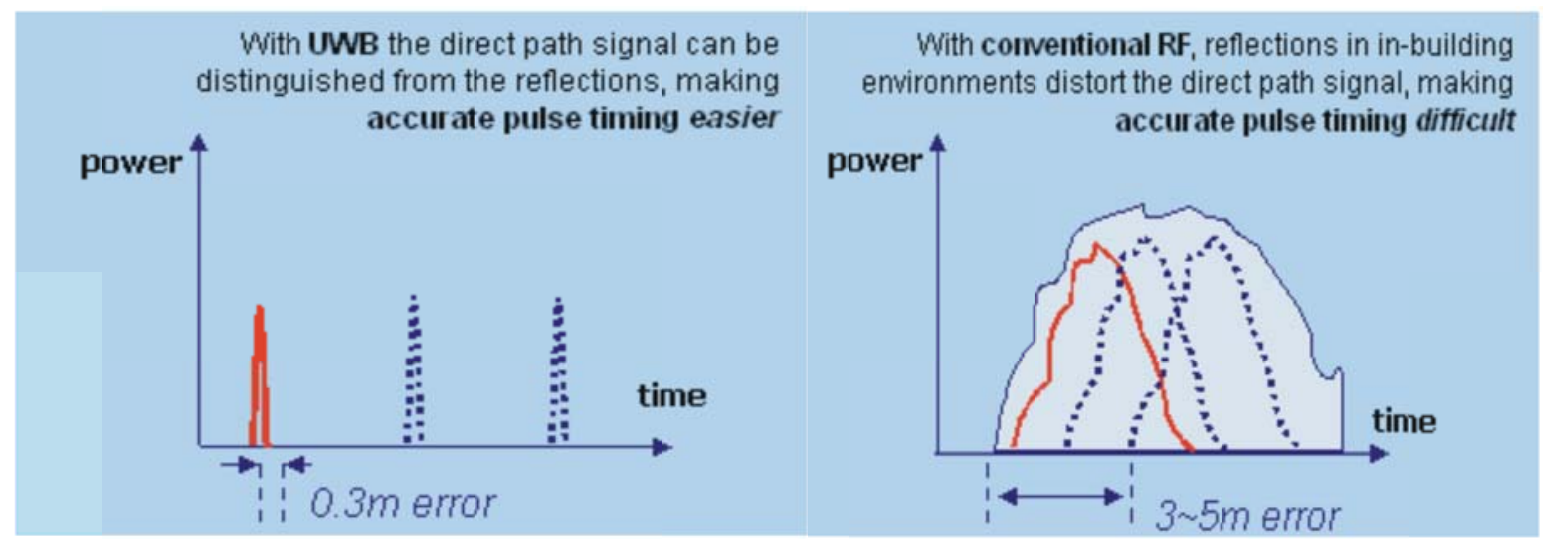

Fig. 1. Advantages of using UWB to positioning - Ubisense

The system consists of immobile receivers (sensors), miniature, mobile transmitters (tags) (Fig. 2, 3) the controlling centre; the most important elements of this centre are the concentrator and the PC computer with Ubisense Location Engine (ULE) software package.
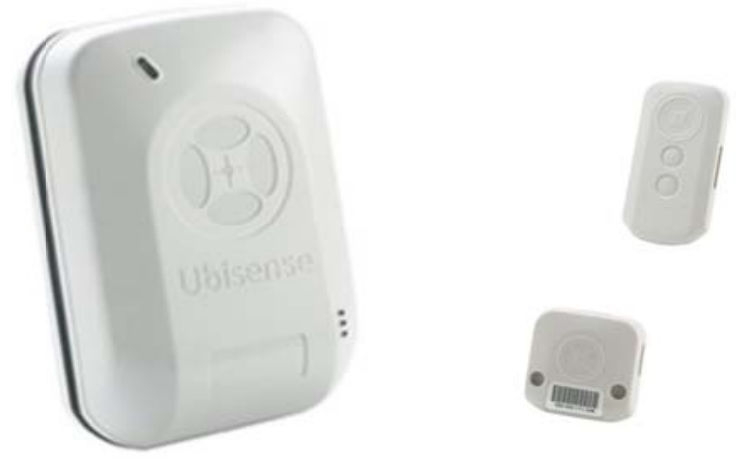

Fig. 2. Ubisense RTLS components - Ubisense Introductionary Workshop

The system allows for simultaneous tracing of many tags. The tag positioning is performed with the frequency up to $10 \mathrm{~Hz}$. The basis for the tag positioning is the combination of two methods: the angular method (AoA - angle of arrival), being a kind of the angular intersection and the pseudorange method (TDoA -time difference of arrival), which delivers information about the difference of the tag distance from particular sensors. 


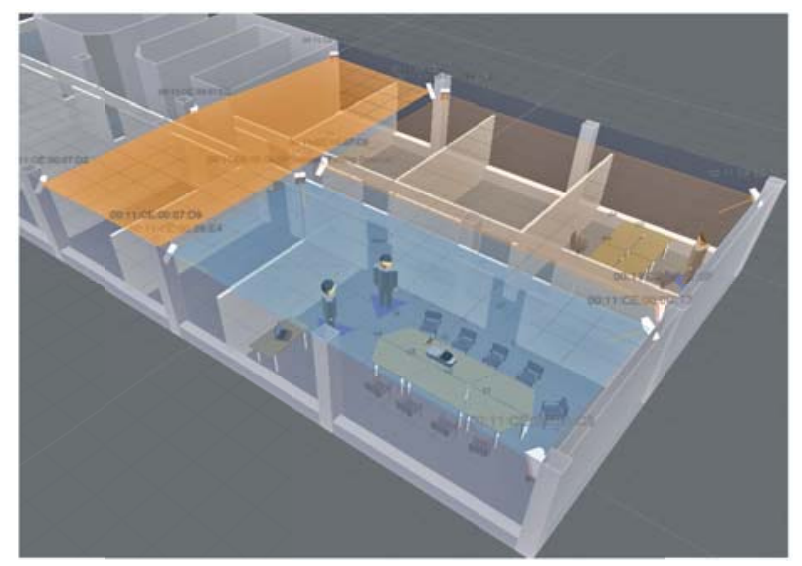

Fig. 3. Typical placement of the sensors - Ubisense Introductionary Workshop

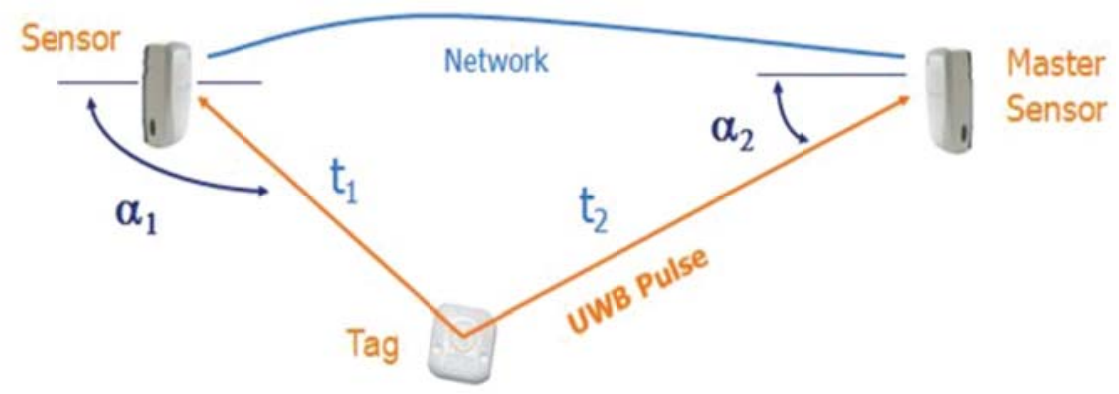

Fig. 4. Idea of determining of tag position - Ubisense

At the moment when the pulse emitted by the tag is received, every sensor determines two angular components of the tag direction. One of the sensors is the master sensor. For every other sensor the system allows for determination of the time difference of the signal arrival, and, as a result, the difference of the distance. Together with angular values, difference of the distance is used for the tag positioning. The general idea of determining of tag position is shown in Fig. 4. The tag position is the geometric location of intersection of appropriate positioning surfaces (Fig.5).

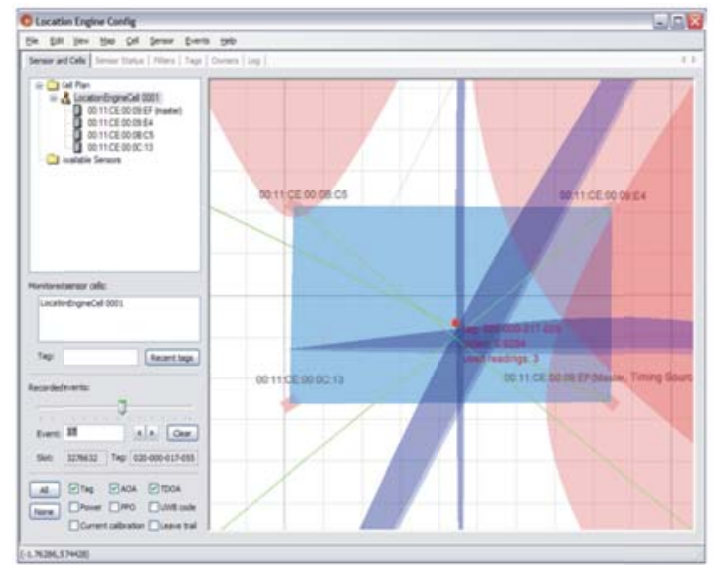

Fig. 5. View of positioning lines and result obtained by Ubisense Location Engine User Manual 


\section{Investigation of theoretical and practical accuracy}

Accuracy of the positioning of Ubisense RTLS were the subject of many investigations described in (Stephan et al, 2009; Gremigni \& Porcino, 2006; Muthukrishnan \& Hazas, 2009; Coyle et al, 2007; Curran et al, 2011; Zhang et al, 2007).

The system accuracy tests performed by the authors were focused on the following three issues:

- testing the accuracy of the system calibration,

- testing the theoretical accuracy of positioning,

- testing the practical accuracy of positioning.

\subsection{Calibration of the system}

After placing the sensors the system calibration should be performed. It aims at determination of the following elements for every sensor:

- spatial position: $X, Y$ and $Z$,

- angles which determine the sensor axes orientation in the assumed coordinate system: Roll, Yaw and Pitch (Fig. 6.).

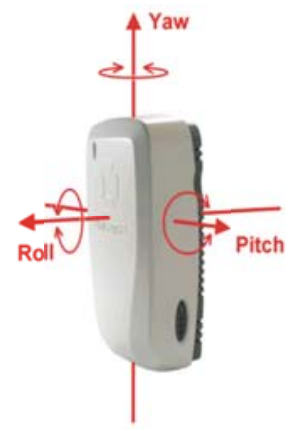

Fig. 6. Sensor's orientation angles - Ubisense

The spatial coordinates, which are required for positioning, are determined with the use of geodetic methods, using an electronic tacheometer. A special procedure of the ULE routine is applied for determination of angular parameters. Following the manufacturer's recommendations (Ubisense, 2008-2009), sensors should be leveled using a level tube. This allows for assuming the null value of the Roll angle (Mandeljc et al, 2010).

Several variants of the calibration procedure exist. Only the simplest variant was accessible in the version of the system, which was tested by the authors; it uses the individual tag position. For this position coordinates should be determined with the use of a total station.

Due to inaccessible internal procedures implemented in the ULE software, testing the calibration accuracy consisted of its multiple repetitions for various tag positions in relation to the stations C4, E6 of the sensors (see Fig. 7). For each position of tag we got the new calibration parameters. 


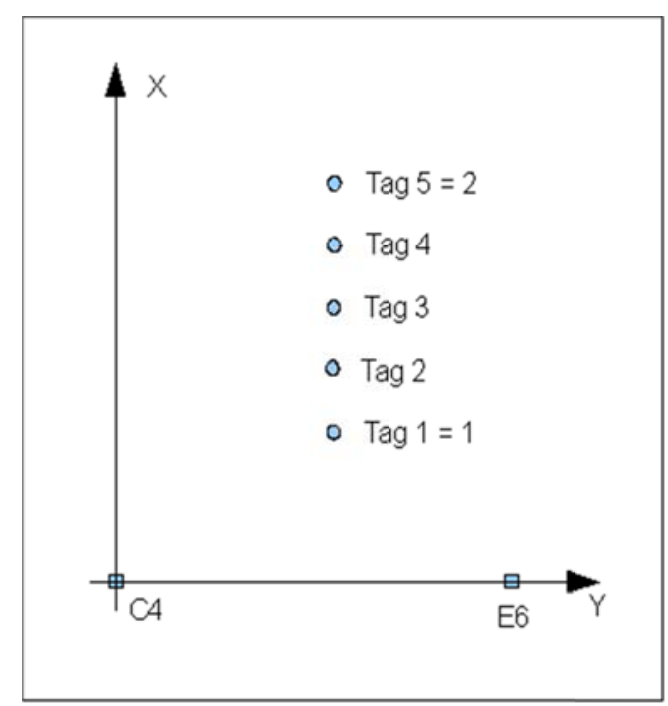

Fig. 7. Configuration of the set used for testing calibration accuracy

The version of the system, which consisted of two sensors, was used for testing. In order to check calibration results, a series of control measurements were performed for each preliminary calibration. They consisted of three-fold determination of two sensors with known coordinates. The parameters of calibration were independently made 3 times for each of five tag positions. Results of test measurements are presented in Table 1.

Table 1. Results of calibration test

\begin{tabular}{|l|l|l|l|l|l|l|l|l|l|l|}
\hline Calibration & $\begin{array}{l}\text { Pitch C4 } \\
\text { grad }\end{array}$ & $\begin{array}{l}\text { YawC4 } \\
\text { grad }\end{array}$ & $\begin{array}{l}\text { PitchE6 } \\
\text { grad }\end{array}$ & $\begin{array}{l}\text { Yaw E6 } \\
\text { grad }\end{array}$ & $\begin{array}{l}\mathbf{X} 1 \\
{[\mathbf{m}]}\end{array}$ & $\begin{array}{l}\text { Y1 } \\
{[\mathrm{m}]}\end{array}$ & $\begin{array}{l}\mathbf{Z 1} \\
{[\mathbf{m}]}\end{array}$ & $\begin{array}{l}\mathbf{X 2} \\
{[\mathbf{m}]}\end{array}$ & $\begin{array}{l}\text { Y2 } \\
{[\mathrm{m}]}\end{array}$ & $\begin{array}{l}\mathbf{Z 2} \\
{[\mathbf{m}]}\end{array}$ \\
\hline Tag 1 & 15,4835 & 45,5165 & 4,30521 & $-26,8684$ & 96,32 & 92,44 & 8,43 & 106,40 & 91,90 & 8,53 \\
\hline & & & & & 96,33 & 92,44 & 8,41 & 105,95 & 91,95 & 8,66 \\
\hline & & & & & 96,30 & 92,42 & 8,42 & 105,92 & 91,95 & 8,89 \\
\hline Std. Dev. & & & & & $\mathbf{0 , 0 2}$ & $\mathbf{0 , 0 1}$ & $\mathbf{0 , 0 1}$ & $\mathbf{0 , 2 7}$ & $\mathbf{0 , 0 3}$ & $\mathbf{0 , 1 8}$ \\
\hline Tag 2 & 11,7249 & 43,8779 & 3,70696 & $-25,9352$ & 96,43 & 92,48 & 8,21 & 107,37 & 92,01 & 7,96 \\
\hline & & & & & 96,45 & 92,45 & 8,27 & 107,31 & 92,00 & 8,00 \\
\hline & & & & & 96,43 & 92,46 & 8,23 & 106,59 & 92,02 & 7,82 \\
\hline Std. Dev. & & & & & $\mathbf{0 , 0 1}$ & $\mathbf{0 , 0 2}$ & $\mathbf{0 , 0 3}$ & $\mathbf{0 , 4 3}$ & $\mathbf{0 , 0 1}$ & $\mathbf{0 , 0 9}$ \\
\hline Tag 3 & 11,1820 & 45,1511 & 2,61231 & $-26,8474$ & 96,32 & 92,55 & 8,17 & 105,66 & 92,37 & 7,84 \\
\hline & & & & & 96,32 & 92,56 & 8,17 & 105,51 & 92,37 & 7,94 \\
\hline & & & & & 96,34 & 92,58 & 8,20 & 105,65 & 92,36 & 8,14 \\
\hline Std. Dev. & & & & & $\mathbf{0 , 0 1}$ & $\mathbf{0 , 0 2}$ & $\mathbf{0 , 0 2}$ & $\mathbf{0 , 0 8}$ & $\mathbf{0 , 0 1}$ & $\mathbf{0 , 1 5}$ \\
\hline Tag 4 & 12,2897 & 45,0506 & 3,82562 & $-26,216$ & 96,36 & 92,59 & 8,24 & 106,11 & 92,33 & 8,14 \\
\hline & & & & & 96,39 & 92,55 & 8,28 & 106,51 & 92,43 & 8,05 \\
\hline & & & & & 96,37 & 92,57 & 8,27 & 106,60 & 92,27 & 8,11 \\
\hline Std. Dev. & & & & & $\mathbf{0 , 0 2}$ & $\mathbf{0 , 0 2}$ & $\mathbf{0 , 0 2}$ & $\mathbf{0 , 2 6}$ & $\mathbf{0 , 0 8}$ & $\mathbf{0 , 0 5}$ \\
\hline Tag 5 & 13,2197 & 43,4716 & 4,13872 & $-25,6608$ & 96,49 & 92,56 & 8,31 & 107,55 & 92,38 & 8,23 \\
\hline & & & & & 96,46 & 92,59 & 8,28 & 106,80 & 92,33 & 8,44 \\
\hline & & & & & 96,48 & 92,58 & 8,30 & 106,60 & 92,34 & 8,31 \\
\hline Std. Dev. & & & & & $\mathbf{0 , 0 2}$ & $\mathbf{0 , 0 2}$ & $\mathbf{0 , 0 2}$ & $\mathbf{0 , 5 0}$ & $\mathbf{0 , 0 3}$ & $\mathbf{0 , 1 1}$ \\
\hline Average & & & & & $\mathbf{9 6 , 3 9}$ & $\mathbf{9 2 , 5 2}$ & $\mathbf{8 , 2 8}$ & $\mathbf{1 0 6 , 4 7}$ & $\mathbf{9 2 , 2 0}$ & $\mathbf{8 , 2 1}$ \\
\hline Std. Dev & & & & & $\mathbf{0 , 0 7}$ & $\mathbf{0 , 0 2}$ & $\mathbf{0 , 0 2}$ & $\mathbf{0 , 5 0}$ & $\mathbf{0 , 0 3}$ & $\mathbf{0 , 1 1}$ \\
\hline
\end{tabular}


The relatively high conformity of determined coordinates of control points No. 1 and 2 may be seen from the above table. The highest variations occur for the $X$ coordinate of point 2. However, the disadvantageous position of this point should be noticed (far from the sensors). Although relatively high variations of the Pitch angle (reaching $4^{\circ}$ ) for the $\mathrm{C} 4$ sensor occur, this component does not have the strong impact on determination of coordinates of control points (variations of the Pitch angle strongly influence the $\mathrm{Z}$ coordinate).

The performed test proved the satisfactory accuracy (repeatability) of calibration parameters, providing that in the case of points of disadvantageous positions, the impact of this procedure considerably exceeds declared accuracy of point positioning, specified by the manufacturer, i.e. $0.15 \mathrm{~m}$ (Liu et al, 20017).

\subsection{Investigation of theoretical accuracy of the system}

The objective of this analysis is to specify the impact of configuration of geometric components of the system on the tag positioning, as well as the accuracy of positioning with the use of a set of receivers, basing on measured angles and differences in distances. The mean errors of coordinate determination, obtained as a result of the analysis, characterize the possible system abilities. The real accuracy will be usually lower due to disturbances of the system operations, mentioned above. Thus, results of performed analyses may be used for interpretation of discrepancies obtained in experimental measurements.

\section{The analyzed area}

In order to present the spatial distribution of estimated values of errors of $X, Y$ and $Z$ determination, the following area was assumed for further considerations:

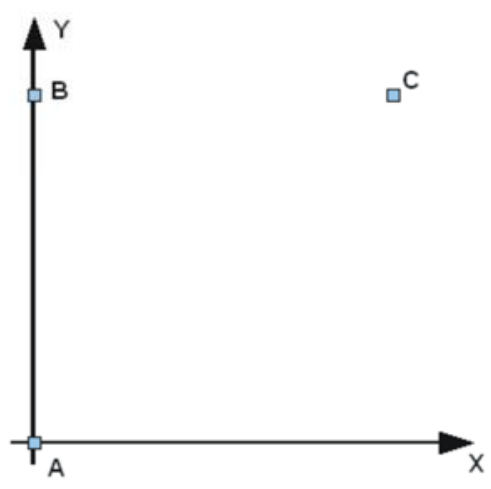

Fig. 8. Sketch of the test field

Positions of receivers are marked as $A, B$ and $C$ (see Fig. 8). The origin of the assumed plain coordinate system is the station $A(X=0, Y=0)$. Other two stations are placed in the corners of the square of the side of $30 \mathrm{~m}$. It was assumed that all receivers are located in the horizontal plane $Z=0$. Besides, the analyzed area was extended by $10 \mathrm{~m}$ in every direction and it creates the square of the side of $50 \mathrm{~m}$. This corresponds to the range of coordinates between $X=-10 \mathrm{~m}$ to $X=40 \mathrm{~m}$, and $Y=-10$ $\mathrm{m}$ to $\mathrm{Y}=40 \mathrm{~m}$. The analysis was performed for two horizontal planes, at the altitudes of $\mathrm{Z}=0 \mathrm{~m}$ and $\mathrm{Z}=10 \mathrm{~m}$. 
Depending on the variant of the analysis, it was assumed that two $(A, b)$ or three $(A, B, C)$ receivers will be used. Due to limitations in extension of angles registered by the sensors, it is not possible to simultaneously determine the tag positions in an arbitrary point of the analyzed area. In the real case, the area possible for determination will become a common part of appropriate sectors, which correspond to particular sensors. The analysis of the area defined in this way results from the fact, that - depending on the sensors' orientation - the common area may be an arbitrary part of the analyzed area.

\section{Accuracy assumptions}

Values, which are measured by receivers in order to determine the tag position, are horizontal angles, vertical angles and differences in distances. In order to evaluate the accuracy of the tag positioning, besides the positions of the tag and the receivers, also the accuracy of measured values should be known. The method of preliminary accuracy analysis, applied by the authors, is based on mean errors. The mean error correspond to the standard deviation value of Gauss distribution for the observation, equated with the random variable.

The documentation, which was made available by the system manufacturer, did not contain information concerning the accuracy of operations performed by the sensor subsystems. More information may be found in accessible scientific papers. The issue of positioning accuracy was the subject of research described by $\mathrm{K}$. Muthukrishnan and Mike Hazas (2010). Results obtained by them differ for various test sites. Besides, considerable diversification of accuracy between horizontal and vertical angles were suggested; however it has not been confirmed by other works and by information presented by the manufacturer. Different values were obtained by Ying Zhang, Kurt Partridge and Jim Reich (2007). They estimated the accuracy of determination of the horizontal and vertical angles as $0.01-0.03$ radian. For the presented work, the direction determination error was assumed as \pm 0.02 radian. The value of $\pm 0.3 \mathrm{~m}$ was assumed as the mean error of the difference of distances.

WALL3D software package was used for calculations. Its basic objective was to develop results of surveys; however, due to its high flexibility it was possible to adapt this package to specific operations of the Ubisense system.

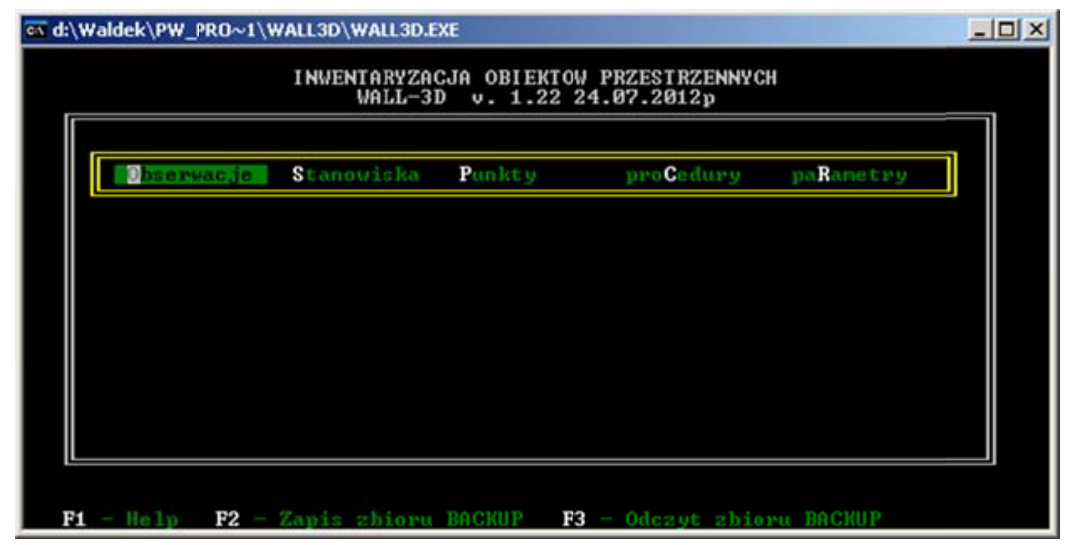

Fig. 9. WALL3D window

The diagrams shown in Fig. $10 \mathrm{a}, \mathrm{b}$ and c present results of the accuracy analysis for particular coordinates, for one of the possible variants. It is the variant with three receivers, for the horizontal plane, $\mathrm{Z}=0$. The role of the "master" sensor is played by the sensor $B$. 
As it may be seen from the diagrams, the accuracy of determination of horizontal coordinates $X$ and $Y$ is satisfactory and it varies within a small interval. Due to the assumed assumptions, the distribution of errors of determination is similar for both coordinates. The "weak" places are areas invisible by the sensors, which are placed in the corners of the analyzed area.

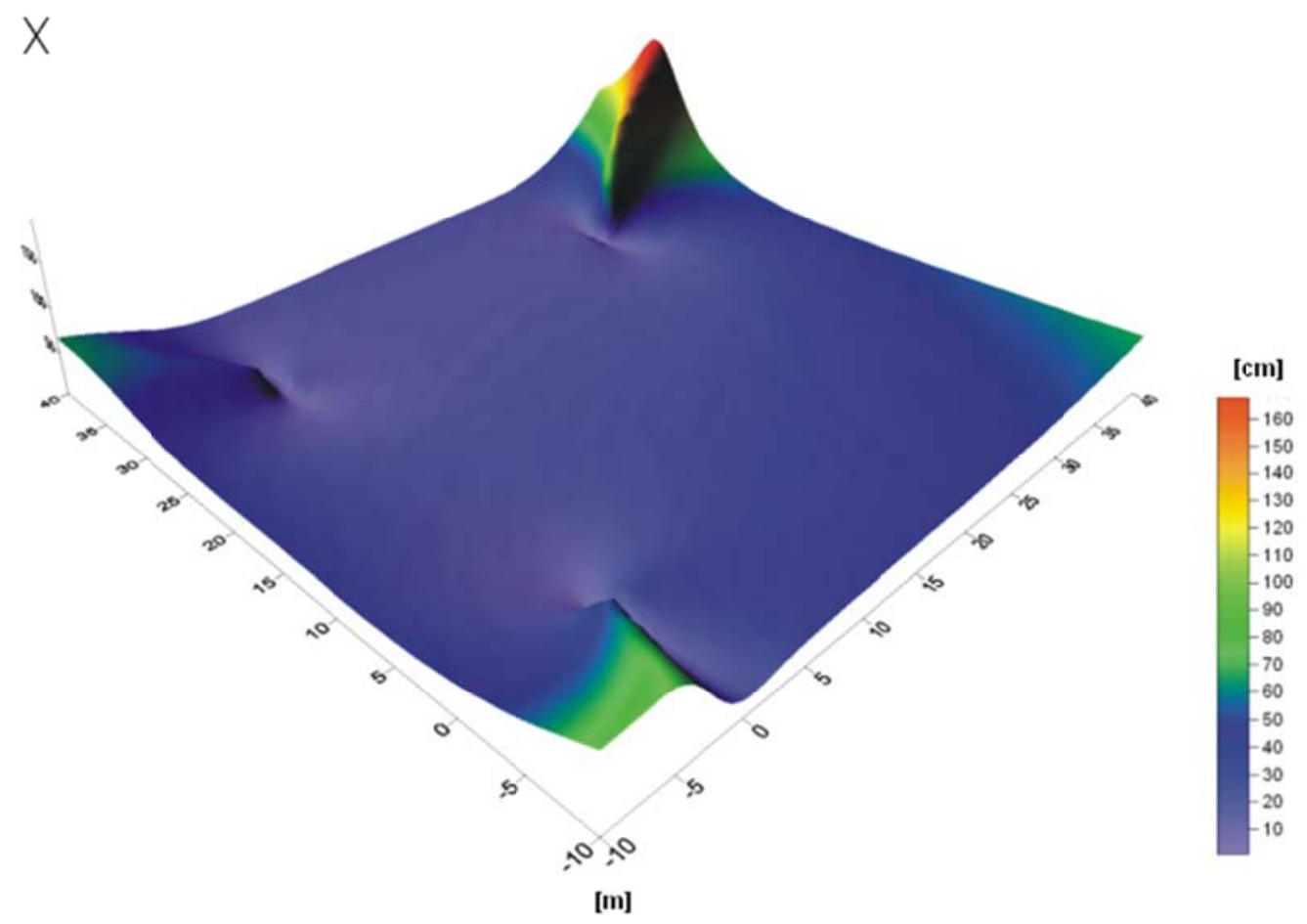

Fig. 10a. Distribution of predicted accuracy of $X$ coordinate

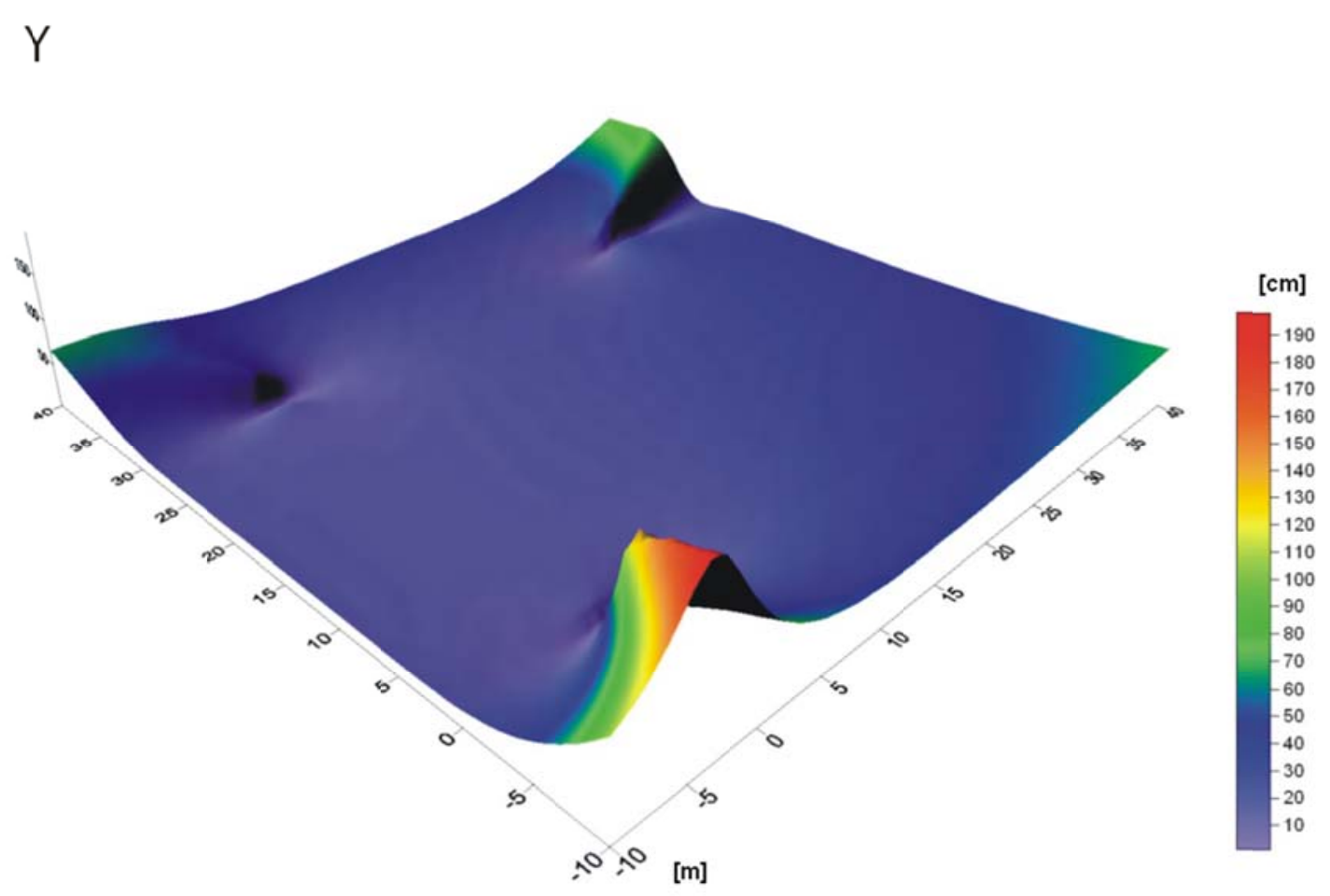

Fig. 10b. Distribution of predicted accuracy of $Y$ coordinate 


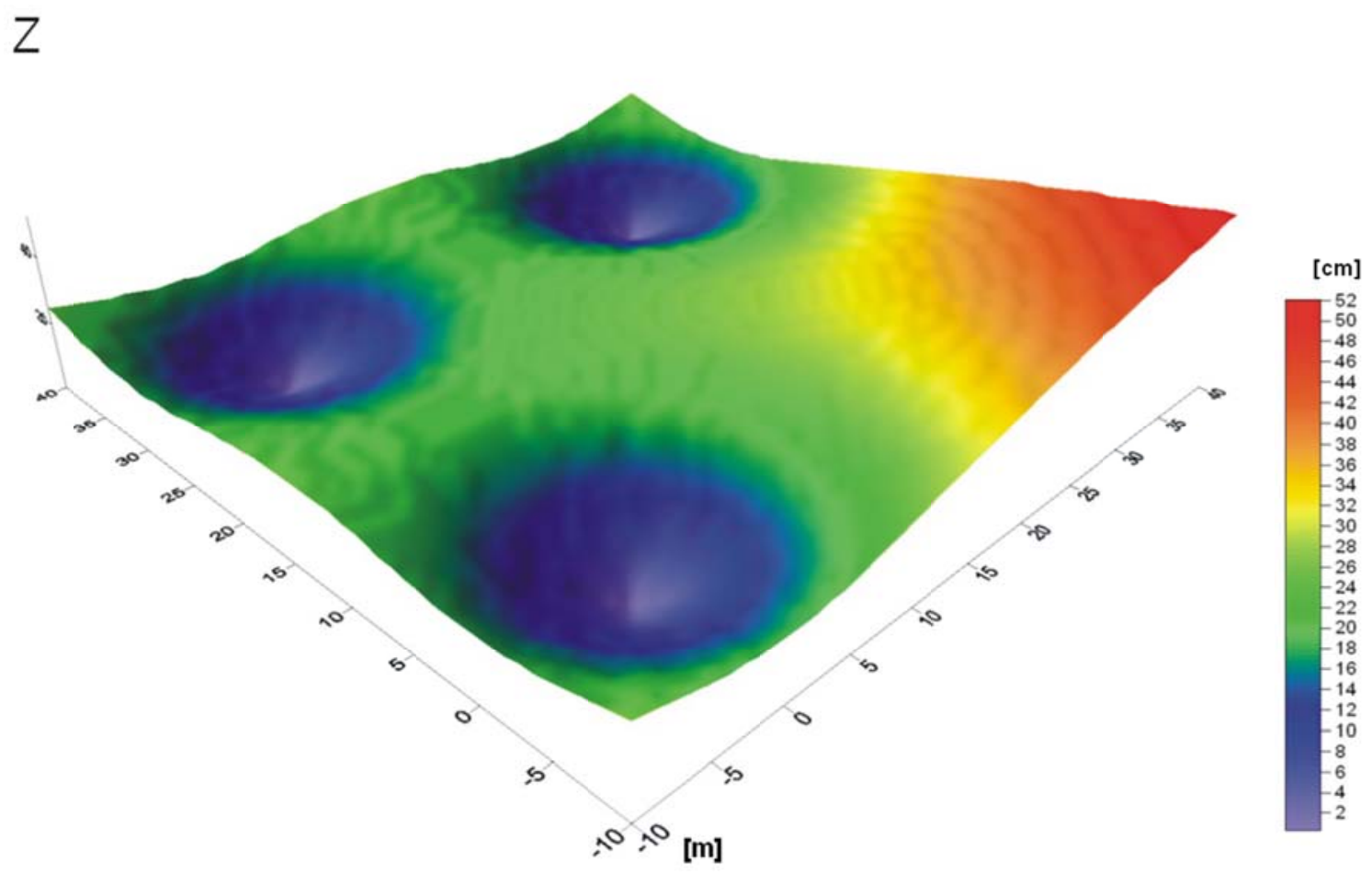

Fig. 10c. Distribution of predicted accuracy of $Z$ coordinate

The characteristics of the distribution of errors for the third coordinate $(Z)$ is slightly different. Those errors are characterized by the higher variability, and the distance between the point and the sensors is the dominating factor which influences the value of the $Z$ coordinate determination error. Regardless this, it should be stated that the $\mathrm{Z}$ coordinate is the coordinate which is determined with the best spatial accuracy.

\subsection{Estimation of real accuracy of the system in the test field}

In order to determine the real accuracy of the Ubisense RTLS system, the measuring test was performed, which covered the entire area of its operations. The floor of the Big Hall of the Warsaw University of Technology was used for testing. The test complied comparison of the tag coordinates, read out from the RTLS system and coordinates measured by means of Leica TCRP 1201+ total station. The same total station was used for determination of sensor coordinates, what ensured the connection between the coordinate systems of the total station and the RTLS system. Each position of the tag of the Ubisense RTLS system was determined three times. The accuracy characteristics of the Leica TCRP 1201+ total station allowed for consideration of differences between coordinates obtained from the Ubisense system and the total station as true errors of the first values. Several tests were performed for various configurations of the sensors.

The distribution of the true errors of $X, Y$ and $Z$ coordinates, obtained from testing, is presented in diagrams (see Fig. $11 \mathrm{a}, \mathrm{b}$ and $\mathrm{c}$ ). 


\section{$x$}
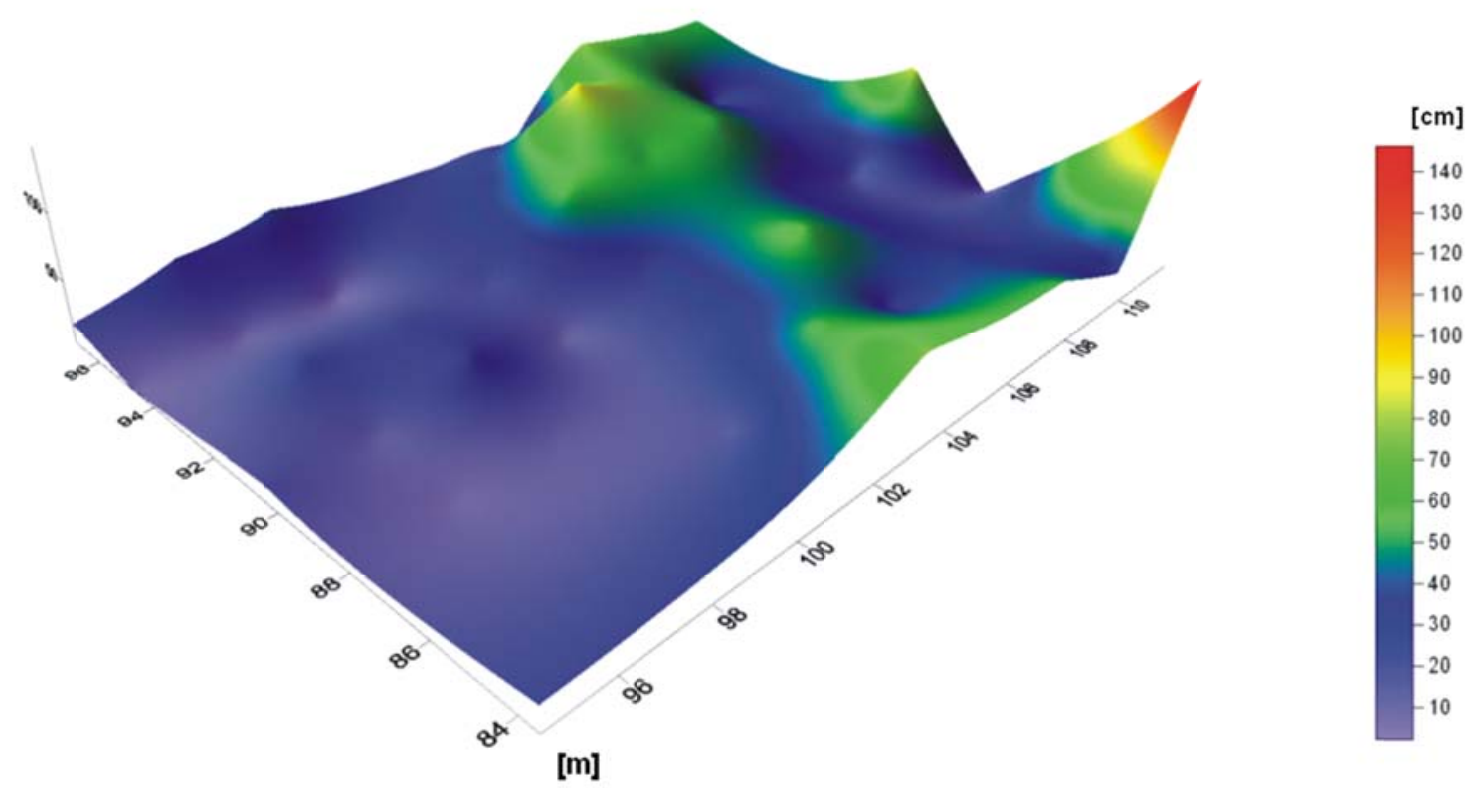

Fig. 11a. Distribution of coordinate $X$ deviation
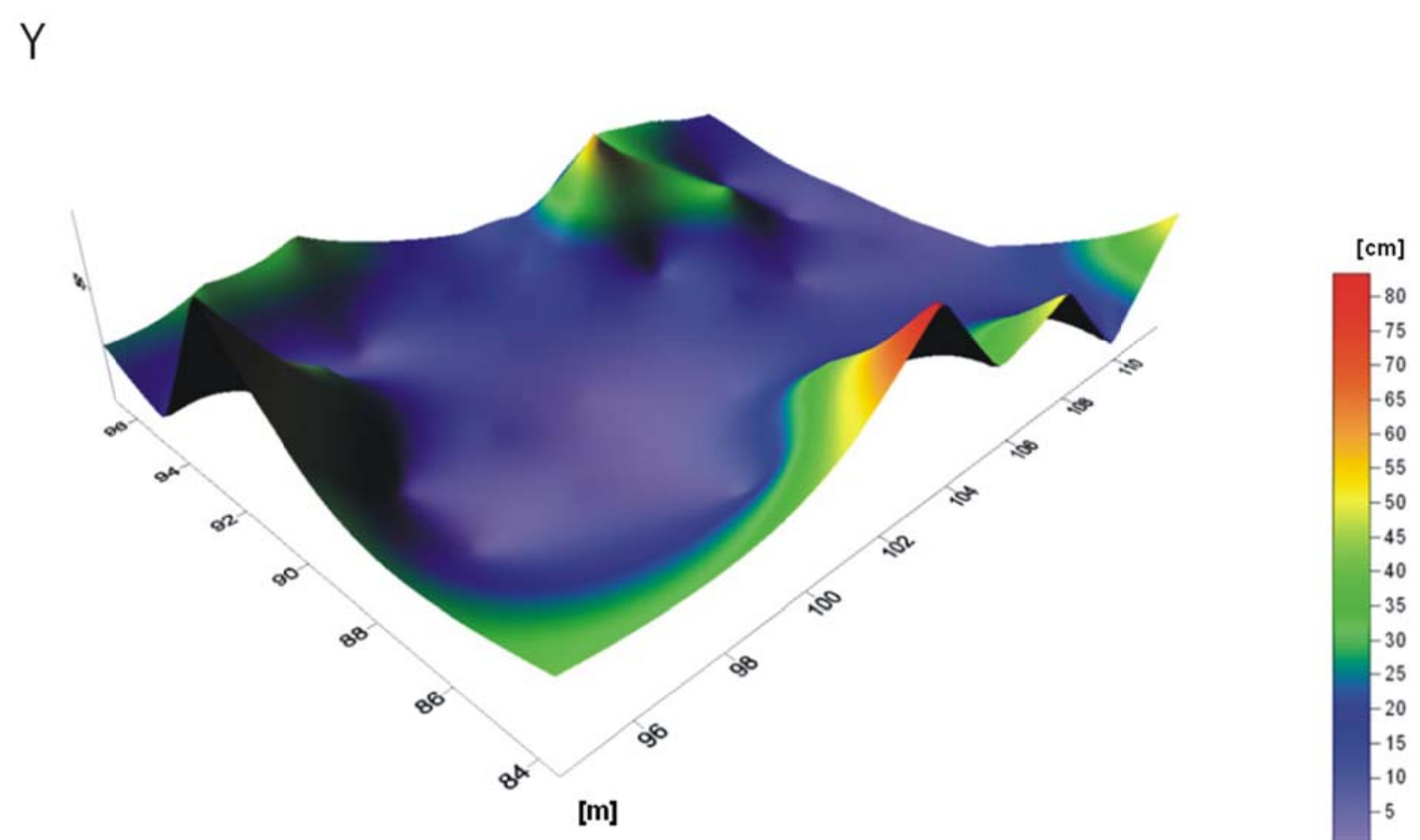

Fig. 11b. Distribution of coordinate $Y$ deviation 

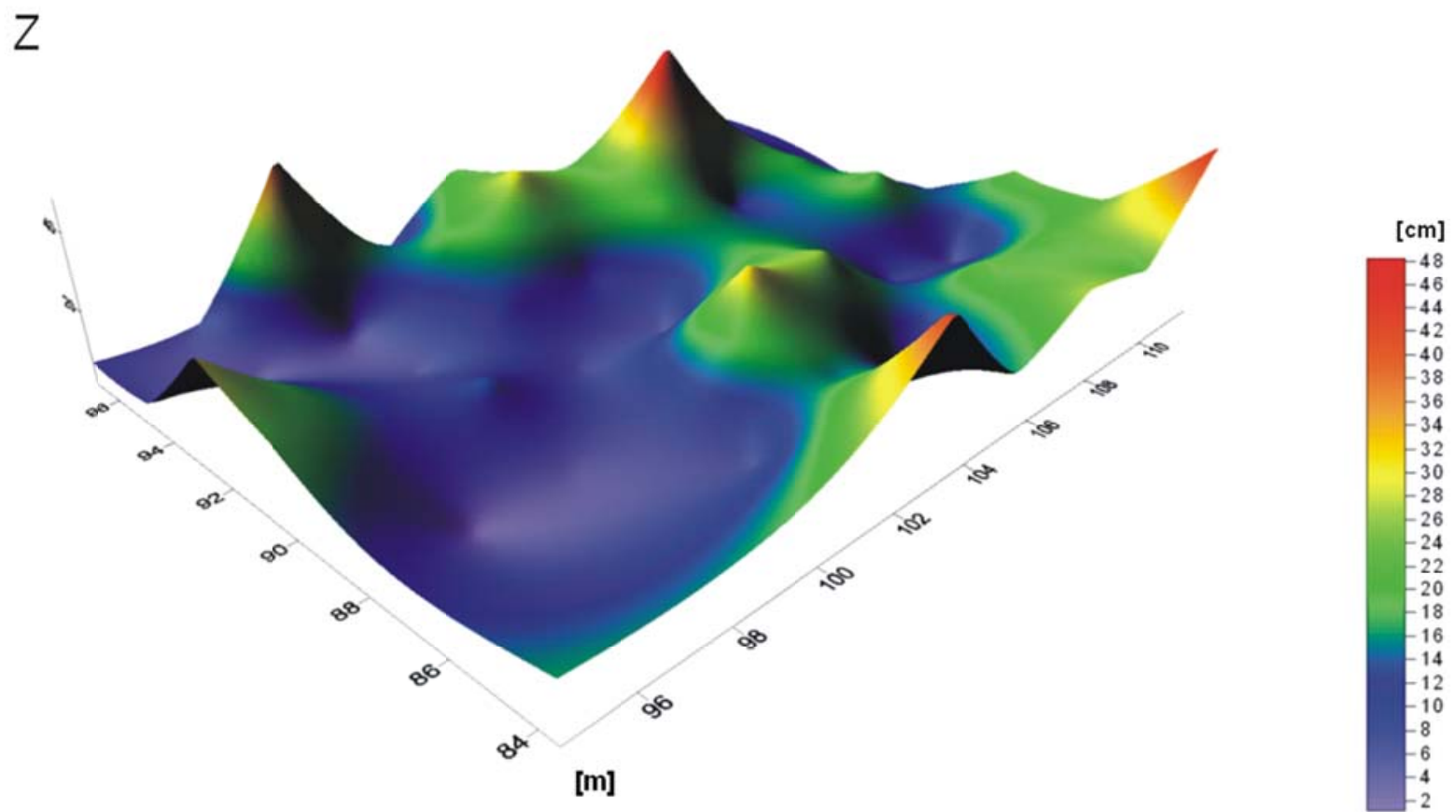

Fig. 11c. Distribution of coordinate $Z$ deviation

As it may be seen, the measuring test confirmed the accuracy values declared by the manufacturer for advantageous geometric conditions. High values of coordinate errors occur at the edges of the tested area and in disadvantageous geometric conditions. The results of test confirm the accuracy forecasts, obtained as a result of the preliminary accuracy analysis, which is presented in section 3.2 of this paper.

\section{Concluding remarks}

Performed analysis and measuring tests allow for formulation of the following final conclusions:

- the preliminary accuracy analysis of the tag positioning may be useful at the stage of planning the placement of the system sensors. This facilitates the achievement of the optimum accuracy in particular conditions and it allows for forecasting critical areas, i.e. areas of lowered accuracy of coordinate determination,

- the analysis of results developed for various configurations of location of the sensor allowed for noticing that the area of the highest accuracy of coordinate determination is the area between the sensors, in particular, when the sensors are mutually turned to each other,

- in order to obtain the optimum accuracy within the entire analyzed area, the sensors should be possibly evenly distributed at the edge of the area, assuming that the increased number of sensors considerably increases the accuracy of reliability of the determined values of their coordinates,

- the system calibration is an important element. Calibration parameters directly influence the determined tag positions. Multiple determination of the sensors' orientation elements should be applied and points, which are the basis for the 
sensors' orientation angles, should be evenly distributed within the "working" area of the system,

- finally it should be stated that both, the results of preliminary accuracy analysis, as well as results of performed measuring tests confirm the accuracy declared by the system manufacturer.

Such systems will be undoubtedly developed, since considerable demands for local navigation systems occurs within areas, which are not visible for satellite navigation systems, as well as for solutions using direct observations.

\section{References}

Coyle, L., Juan, Y., Loureiro, E., Knox, S., Dobson, S. \& Nixon, P. (2007). A Proposed Approach to Evaluate the Accuracy of Tag-based Location Systems, Ubiquitous Systems Evaluation (USE 2007). Workshop at UbiComp2007, pp. 292-296, 2007

Curran, K., Furey, E., Lunney, T., Santos, J., Woods, D. \& Mc Caughey, A. (2011). An Evaluation of Indoor Location Determination Technologies, Journal of Location Based Service Volume 5, Issue 2, 2011

Gremigni, O. \& Porcino, D. (2006). UWB ranging performance tests in different radio environments, London Communications Symposium 2006

Stephan, P., Heck, I., Kraus, P. \& Frey, G. (2009). Evaluation of Indoor Positioning Technologies under industrial application conditions in the SmartFactory based on EN ISO 9283, Preprints of the 13th IFAC Symposium on Information Control Problems in Manufacturing, Moscow Russia June 3 - 5, 2009

Liu, H., Darabi, H., Banerjee, P. \& Liu, J. (2007). Survey of Wireless Indoor Positioning Techniques and Systems, Systems, Man, and Cybernetics, Part C: Applications and Reviews, IEEE Transactions, vol. 37, issue: 6, November 2007

Mandeljc, R., Perš, J., Kristan, M. \& Kovačič, S. (2010). An Alternative Way to Calibrate Ubisense Real-Time Location System via Multi-Camera Calibration Methods. Proceedings of the 19th International Electrotechnical and Computer Science Conference, ERK 2010, pp. 249-252

Muthukrishnan K. \& Hazas, M. (2009). Position Estimation from UWB Pseudorange and Angle-of-Arrival: A Comparison of Non-linear Regression and Kalman Filtering, Proc., 4th Int. Symp. on Location and Context Awareness, Springer, pp. 222-239

\section{Ubisense (2008-2009). LocationEngineConfig User Manual}

Ward, A., Webster, P. \& Batty, P. (2003). Local Positioning Systems - technology overview and applications, Ubisense White Paper, September 2003

Zhang, Y., Partridge, K. \& Reich, J. (2007). Localizing Tags Using Mobile Infrastructure, Location- and Context-Awareness, Lecture Notes in Computer Science Volume 4718, pp. 279-296 


\section{Authors:}

Assoc. Prof. Marek Woźniak ${ }^{1)}$, m.wozniak@gik.pw.edu.pl

PhD Waldemar Odziemczyk ${ }^{1)}$, w.odziemczyk@gik.pw.edu.pl Msc Kamil Nagórski ${ }^{1)}$

1) Warsaw University of Technology, Faculty of Geodesy and Cartography,

PI. Politechniki 1, 00-661 Warsaw, Poland 